\title{
An anti-collision algorithm of RFID reader
}

\author{
Lan Hai-yan \\ College of computer science and technology \\ Harbin Engineering University \\ Harbin 150001, china \\ Lanhaiyan@ hrbeu.edu.cn \\ Zhao Yun-long \\ College of computer science and technology \\ Harbin Engineering University \\ Harbin 150001,china \\ ZhaoYunlong@hrbeu.edu.cn
}

\author{
Song Hong-tao \\ College of computer science and technology \\ Harbin Engineering University \\ Harbin 150001, china \\ Songhongtao@hrbeu.edu.cn \\ Zhang Guo-yin \\ College of computer science and technology \\ Harbin Engineering University \\ Harbin 150001,china \\ Zhangguoyin@hrbeu.edu.cn
}

\begin{abstract}
For interference problems between multiple readers in the RFID (radio frequency identification) system of the Internet of Things, an anti-collision algorithm of RFID reader (CAAC) based on cultural optimization is proposed. The reader starts hierarchical clustering in the RFID network, by dynamically adjusting the signal power range of the reader, so that each reader using different transmission power in the work within the same time slot. Therefore, the overlapping area between the reader is reduced to obtain the maximum system throughput. Cultural algorithm (CA) is adopted to search for optimal power allocation scheme, making the search more efficient and meanwhile, goal optimization and variable optimization is likely to get numerical quantification. Simulation shows that the proposed anti-collision algorithm of RFID improves the running time of the reading and reduces the energy consumption and interference between readers.
\end{abstract}

Keywords-Internet of things ; RFID ; Anti-collision ; Cultural algorithm

\section{INTRODUCTION}

Internet of Things, as one of the seven strategic emerging industries in our country ${ }^{[1]}$, has broad prospects for development. RFID technology, the core of Internet of Things, has been widely used. RFID system is generally made by electronic tags (Tag) Reader (Reader) and the host processing system, which uses RF signal transmission characteristics and spatial coupling (inductive or electromagnetic coupling) to automatically identify the target objects. Like the wireless communication system, there also exists the problem of signal interference in the RFID system.

Signal interference is mainly divided into two kinds: one kind is called reader collision, which refers to the adjacent reader mutual interference in the signal superposition region, causing the label in the region not to response to any reader; Another kind is the tag collisions, which means that collision is introduced when multiple tags reply a reader at the same time, the reader cannot normally identify tag information. Signal collision is the key to influence the performance of radio frequency identification ${ }^{[2-4]}$. Therefore, reader and tag collision prevention protocol is needed to reduce reader collision and tag collision, so that the performance of the identification process can be improved.

\section{THE PREVENTION OF RFID READER COLLISION}

The reader with overlapping region might have interference with each other in the label recognition. Similar to other RF system, even if the recognition region is not overlapped, a reader may also affect the operation of the other reader, leading to the reader collision. Due to the fact that energy of label is provided by the reader, the response area of the label is smaller than the sending area. As shown in figure 1 , the when a tag $\mathrm{T} 1$ is within the recognition area of reader R1 and is within interference zone of the reader R2, due to the interference between the readers, the tag cannot correctly receive a request from the reader R1 command, or reader $\mathrm{R}$ cannot correctly receive response from the label, defined as the reader collision.

There are many methods to solve the problem of reader collision, such as the mechanism based on scheduling, power adjusting and learning.

In the scheduling scheme, the frequency and time slot are scheduled for the reader. Scheduling can be centralized or distributed, and support the static and dynamic frequency distribution. Waldrop proposed two algorithms, color selection algorithm DCS and Colorwave respectively, which are typically TDMA reader collision prevention protocol ${ }^{[5]}$. In addition, the methods based on learning is online learning based on hierarchy, genetic algorithm and neural network ${ }^{[6]}$. Learning methods minimize collision by learning collision model and distributing frequency based on learning mode, such as HiQ algorithm ${ }^{[7]}$. HiQ protocol is a distributed, classification, and online learning algorithm, designed to achieve the goal of maximizing concurrent communication channels between the reader and the tag and through the learning of the reader collision model to minimize the number of reader collision, so that time slot is effectively allocated to the reader. Beacon (beacon) channel ${ }^{[8]}$ and the center coordinator, as well as alternative method coping collision as covering, collision such alternate method have 
scheduling scheme that in the whole network system structure, reader collision messages are uniformly collected and the available resources are reasonably allocated to each reader in order to prevent interference caused by multiple reader sending signals to label at the same time. Lots of resources are required to establish and maintain a whole structure. When there is a slight change in the system, a whole range of resource allocation should be adjusted, introducing high system cost.

Power adjustment makes every reader use different sending power at the same time in the work through adjusting the signal power range of the reader so as to reduce the overlapping area between the readers and obtain the maximum range of reading. The representative agreements are LLCR and w - LCR ${ }^{9]}$. This algorithm is not only complex and can lead to inefficiency and the reduction of power consumption, but also need to the introduction of the new control equipment . The system cost is higher. Although existing algorithms have set up a file in the wide application of radio frequency identification , there are such drawbacks as high power consumption and high cost. In this paper reader collision prevention algorithm based on cultural algorithm is proposed aiming at power adjustment.

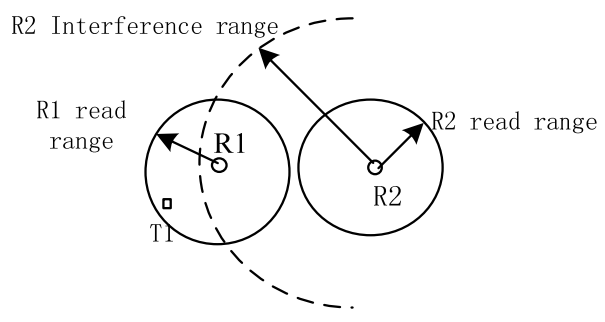

Figure 1. Reader and reader interference

\section{THE COLLISION PREVENTION ALGORITHM BASED ON CULTURAL ALGORITHM}

This algorithm is based on power adjustment, using the cultural algorithm to optimize the reader transmission power, so as to realize effect on the throughput of the system resulted from reader collision communication in transmission phase

\section{A. Cultural Algorithm}

Reynolds put forward a new evolutionary algorithm cultural algorithm (CA) for the first time in $1994^{\text {[10] }}$, which gained knowledge to solve the problem from evolutionary population (religion), and these knowledge will be used to guide the search process. The double evolutionary system structure based on knowledge of culture algorithm makes the population increase and adapt to the environment so that it shows better performance of global optimization in the search process. Culture algorithm framework provides the calculation model of multiple evolutionary processes, and a kind of any evolutionary algorithms, as a process of evolution in population space, which can meet the requirements of the culture algorithm can be embedded in the framework of cultural algorithm. Cultural algorithm based on the traditional evolutionary algorithm extracts beliefs guiding the search space, which makes the search efficiency higher. And meanwhile optimization of objectives and optimization of variables are relatively easy to numerical quantification. Although CA algorithm is complex, its computing time is greatly reduced ${ }^{[1]}$.

\section{B. Optimization Model}

When more than one reader participate in tag identification, the reader collision problem occurs, so that how to effectively allocate resources in the RFID system of limited energy consumption for the sake of improving the throughput of the system and identifying efficiency has very great significance. Assuming that the number of the reader in the RFID system is $N$, the optimizing objective is to maximize the system throughput. The throughput refers to the data amount within a predetermined time from a transmitting end to the receiving end. The definition of the fitness function is as follows:

$$
\operatorname{argmax} S=\frac{\sum_{i=1}^{N_{\text {tag }}} R_{i}}{T}
$$

$$
\begin{gathered}
\text { Constraints: } \sum_{j=1}^{N_{c}} b_{i, j}=R_{i} \\
\text { SIR }_{i} \geq S I R_{\text {targ et }}
\end{gathered}
$$

Where, $N_{\text {tag }}$ is the number of labels successfully read, $R_{i}$ is successfully transmission rate for the first time, $\mathrm{T}$ is the sampling period, $S I R_{i}$ is the limited by the signal to interference ratio $S I R_{\text {targ et }}$.

\section{Algorithm Steps}

The key of using cultural algorithm to solve the resource allocation problem of RFID system lies in the decoding of parameters and settlement of the fitness function. Specific implementation steps are as follows:

1.The initial population. Population space adopts evolutionary programming population model. Individual coding method uses real number coding. An individual is formulated as:

$$
X=\left[x_{1}, x_{2}, \cdots, x_{N}\right]
$$

In equation (2), $\mathrm{N}$ is the number of readers, and $x_{1}, x_{2}, \cdots, x_{N}$ settled transmission power. Firstly, simple operation of the power allocation (not the best) is implemented to make each labels meet the requirements of the recognition rate. Within the scope of bit constraint, a $\mathrm{N}$ dimensional real vector is randomly generated, so that has an individual in the space. Repeat the above steps $p$ times and produce the population scale for initial population p space. 
2. Settlement of the fitness function. Evaluate the individual in population space. The greater the objectives function, the more resource allocation scheme can meet the design index. This is a problem to maximize system throughput. Defined (1) is the fitness function.

3. According to the given value range, the candidate solutions of initial population space and the belief space structure, initial belief space is generated. The structure of belief space uses the pair of $\langle S, N\rangle$ in which $S=\left\{s_{1}^{t}, s_{2}^{t}, \cdots, s_{m}^{t}\right\}$ is the knowledge, representing the optimal individual collection.

4. According to the influence function, every father individual in the population spatial varies, generating corresponding individual. The knowledge is used to adjust the change step length of variables and the situation knowledge adjusts its direction.

5. For the population space made by the son and the father individuals, the scale is $2 p$. $c$ individuals are randomly selected from the population space. If the individual is superior to the comparison individual , this individual gains a victory, and victory number of each individual is recorded. Choice $p$ individuals with the most number of victory as father of the next generation.

6. Setting accept function and updating the belief space ${ }^{[12]}$. Accept function is used to choose individual directly affecting the knowledge and experience of the current belief space. Based on the current population, the optimal proportion is chosen at ration $\beta$, namely accept $=[p \cdot \beta]$.

7. Turning to step 4 until to the limit or already convergence to termination conditions, namely error reaching the setting value or iteration times exceeding maximum times and stop searching. The global optimal combination is output as the optimal scheme of reader power allocation.

\section{SimUlATION ANALYSIS}

In order to test the cultural algorithm on resource allocation in terms of reader anti-collision performance, CAAC algorithm is compared with colorwave ${ }^{[5]}, \mathrm{HiQ}^{[7]}$ and LLCR $^{[9]}$. In the matlab platform through 100 times of Monte Carlo simulation, the label uses ISO/IEC18000-6C agreement, reader antennas are omni-directional antenna, reader number is as $\mathrm{N}=[3-30]$, all readers are placed randomly and the tag number is 100 . Initial population is completely randomly generated. The initial population size is 10, competitive scale are $C=10, \beta=0.16$, crossover probability is 0.63 and mutation probability is 0.02 .

Figures 2 and 3 give the various throughput of various algorithms, the relationship between system efficiency and the number of readers. It can be seen that CAAC algorithm is better in the throughput and efficiency which demonstrates the feasibility and effectiveness of anti-collision algorithm. As shown in figure 2, figure 3 and figure 4, in condition of
$\mathrm{N}=4 、 \mathrm{~N}=10$ and $\mathrm{N}=20$, the system energy consumption i.e. reader transmit power performance is analyzed. When the number of users is small, colorwave algorithm has better performance. With the number of readers increased to $\mathrm{N}=10$, the obvious performance advantages of the proposed algorithm are not very great. When $\mathrm{N}$ is increased by 20 , as shown in figure 6, CAAC algorithm shows better anticollision performance. Thus, with the increase of the number of readers, the advantages of the CAAC algorithm are larger. Simulation results show that the proposed anti-collision algorithm performs better on system throughput, system efficiency and energy consumption.

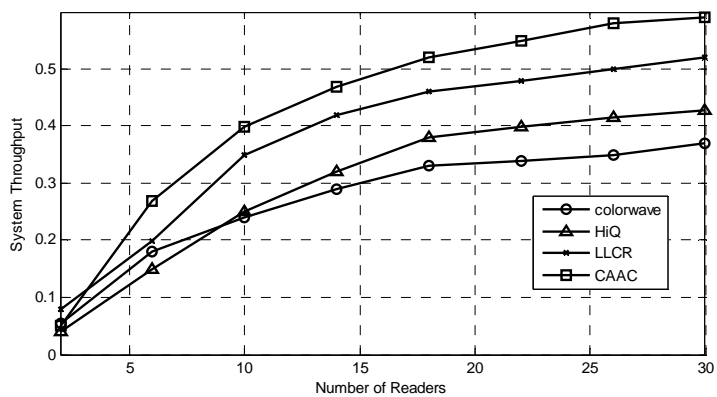

Figure 2. System Throughput of the algorithms

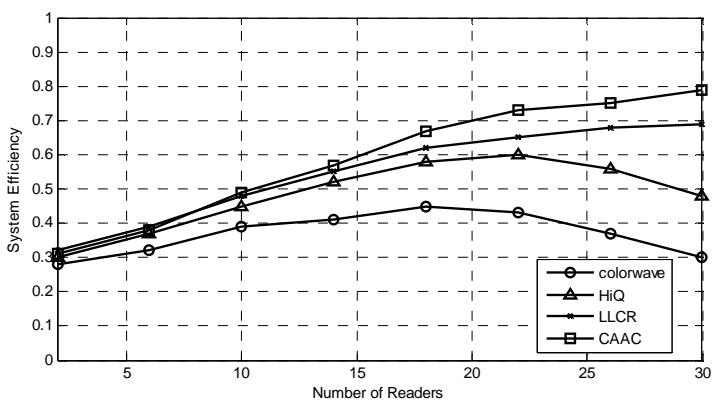

Figure 3. System Efficiency of the algorithms

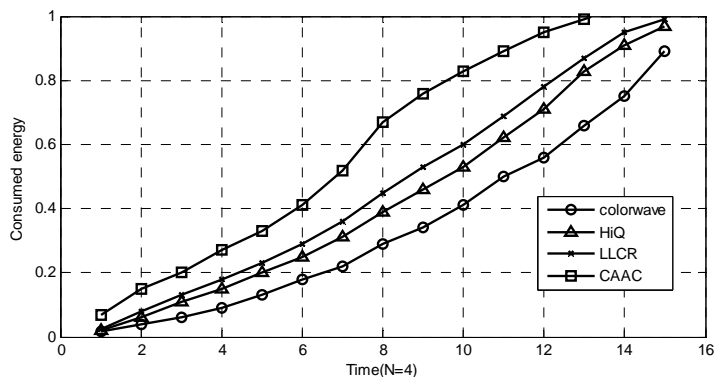

Figure 4. Consumed energy of the algorithms $(\mathrm{N}=4)$ 


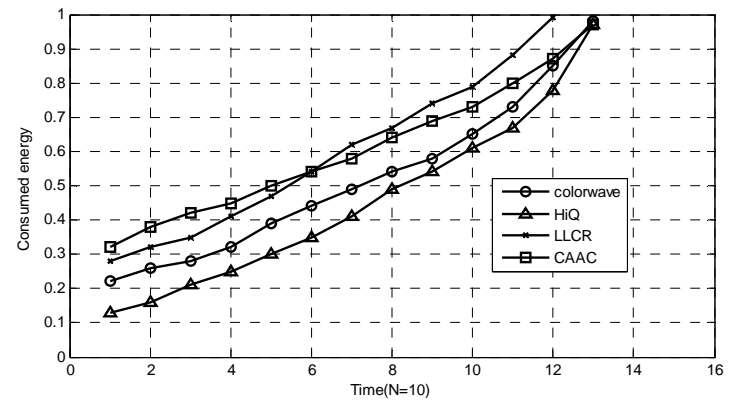

Figure 5. Consumed energy of the algorithms $(\mathrm{N}=10)$

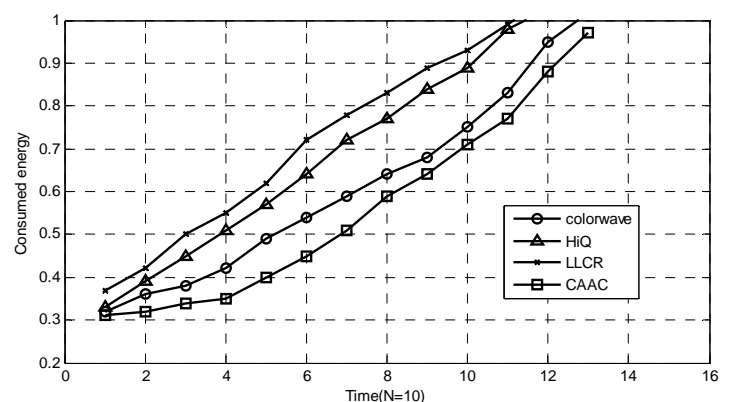

Figure 6. Consumed energy of the algorithms $(\mathrm{N}=20)$

\section{CONCLUSION}

Based power adjustment, a multi-reader anti-collision algorithm is proposed. Cultural algorithm is used in the search for the optimal RFID in terms of the multi-reader collision problem, which makes it more concentrated and quickly close to the optimal solution, so that better distribution scheme is obtained. This algorithm provides a theoretical basis for the next generation of electronic tags and readers in the Internet of Things.

\section{ACKNOWLEDGMENT}

This work was supported by Postdoctoral Foundation of Heilongjiang province under Grant No.LBH-Z10188,
Fundamental research funds for the central universities under Grant No .HEUCFR1014, HEUCFZ1105, and the National Natural Science Foundation of China under Grant No.61073042, Harbin Scientific \& Technological Innovation Research Funds under Grant No. 2011RFQXG012

\section{REFERENCES}

[1] ATZORI L,IERA A,MORABITO G.The internet of things:a survey[J].Computer Networks,2010,54(15):2787-2805.

[2] J.Myung,W.Lee, and J.Srivastava,Adaptive binary splitting for efficient RFID tag anti-collision,IEEE Communications Letters, 10,144-146,2006.

[3] S.H.Kim and P.Park,An efficient tree-based tag anti-collision protocol for RFID systems,IEEE Communications Letters,11,449-451,2007.

[4] EPCglobal.EPCglobal Class-1 generation-2 UHF RFID protocol,April 2004.Version 1.0.9

[5] J.Waldrop,D.W.Engels, and S.E.Sarma.Colorwave:An anti-collision algorithm for the reader collision problem.In proc.of IEEE international conference on communications,vol 2,pp.12061210,Anchorage,Alaska,2003.

[6] C.Lin,F.Lin,A simulated annealing algorithm for RFID reader networks,WCNC'2007:IEEE Wireless Communications and Networking Conference,pp.1669-1672,Hongkong,China,2007.

[7] J.Ho,D.W.Engels,S.E,Sarma,HiQ:A hierarchical Q-learing algorithm to solve the reader collision problem,SAINTWorkshops 2006:International symposium on Applications and the Internet Workshops,pp.88-91,Phoenix,AZ,2006.

[8] S.M.Birari and S.Iyer,Mitigating the reader collision problem in RFID networks with mobile readers,13th IEEE International Conference on Networks,pp.463-468,Kuala Lumpur,Malaysia,2005.

[9] J.Kim,J.Yu,J.Myung,and E.Kim,Effect of localized optimal clustering for reader anti-collision in RFID networks:Fairness aspects to the readers,ICCCN 2005:14th International Conference on Computer communications and Networks,pp.497-502,San Diego,CA,2005.

[10] R.G.Reynolds and D.B.Fogel.Proceedings of the Fourth Annual Conference on Evolutionary Programming, MIT Press, Cambridge, Massachusetts, 1995: 298-305P

[11] D.B.Fogel . An introduction to simulated evolutionary optimization. IEEE trans. on neural networks,1994, 5(1): 3-14P

[12] Lan H Y,Yang S Y,Liu H B,MA X S.Resource allocation for multiuser OFDM system based on cultural algorithm.Journal of Jilin University(Engineering and Technology Edition),vol 41, No 1,Jan 2011,pp 226-230 